\title{
Actitudes de los estudiantes de la Universidad Tecnológica Equinoccial hacia el emprendimiento, Quito, Ecuador, Octubre del 2007
}

Autores:

Dr. Gilberto Vejarano Ing. Pilar Espinosa 


\section{Resumen}

Los objetivos del estudio fueron, identificar y medir la actitud emprendedora de los estudiantes de la UTE del Campus Quito, para diseñar estrategias de promoción, comunicación, capacitación e impulso al emprendimiento, para lograr establecer las estrategias en estos aspectos y lograr mantener la excelencia. Un total 6.250 estudiantes de las tres jornadas conformaron la población de estudio.. Se seleccionaron a través de un muestreo no Probabilístico, intencionado proporcional al tamaño de las Facultades y Programas Académicos. Para el caso de que el número de la muestra fuera 0 se decidió tomar 5 casos para que la Escuela respectiva no quedara eliminada del estudio; un total de 245 estudiantes quedaron incluidos en la muestra. Se utilizó el Cuestionario de Auto Evaluación de las conductas emprendedoras, desarrollado y probado por el Programa Formación de Multiplicadores en Emprendimiento de INCUBATEC (Incubadora de Negocios de Temuco, Chile). Los resultados demostraron que existen actitudes y conductas altamente positivas hacia el emprendimiento, así como de apoyo a las estrategias que conlleven a fortalecer las actividades que conduzcan a fomentar una cultura emprendedora. Un perfil, de acuerdo a los valores de los indicadores del Test utilizado, altamente positivo entre los estudiantes tanto de uno como en otro género. Se puede inferir que la Universidad está acertando en sus estrategias hacia un enfoque de formación académica que conlleve al emprendimiento en sus estudiantes y motivación en los docentes.

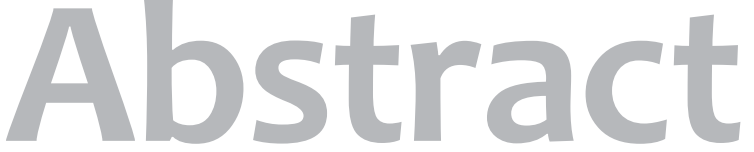

The objectives of the study was to identify and measure the entrepreneurial attitude of the students in the Campus UTE Quito, to design strategies for advocacy, communication, training and promoting entrepreneurship, establishing strategies to achieve in these areas to maintain and reach excellence. The study population was constituted by a total 6250 students which are part of the three studying schedules. This population was selected through a nonprobability sampling that is proportional to the size of the faculties and academic programs. In the event that the sample number was 0 it was decided to take 5 cases were decided 5 so that the faculty would not be eliminated from the study; a total of 245 students were included in the sample. We used the Self-Assessment Questionnaire of entrepreneurial behavior, developed and tested by the Program in Entrepreneurship Training of Multipliers INCUBATEC (Business Incubator of Temuco, Chile). The results showed that there were highly positive attitudes and behaviors towards entrepreneurship and to support strategies that involve strengthening activities that lead to foster an entrepreneurial culture. A profile, according to the values of the test used, shows a highly positive towards this culture among both male and female students. It can be inferred that the University is becoming successful in its strategies toward a focus of academic training that leads to entrepreneurship in their students' motivation and teachers. 


\section{Introducción}

El factor emprendedor está presente en todas las acciones humanas (Ludwing von Mises); al hablar de emprendimiento surgen ideas como innovación, creatividad, liderazgo, empresa, en definitiva lo consideran como un proceso dinámico de formación y desarrollo de empresas, ya sea porque el emprendedor está alerta a oportunidades que existen en situaciones inciertas (Escuela Austriaca) o porque genera desequilibrios en los mercados a través de la introducción de innovaciones (Shumpeter), quien manifiesta también que además de las capacidades técnicas y experiencia, el ejercicio de la intuición y estrategia es de particular importancia debido a la incertidumbre inherente al proceso de desarrollo.

En la práctica es probable que ambos modos de emprendimiento co-existan en una persona o una sociedad para favorecer el desarrollo; no está ligado al número de empresas creadas sino a la facultad de crecer y de crear riqueza, está orientado al crecimiento, al encaje de tres puntos básicos: mercado, personas y recursos.

De acuerdo a varios estudios realizados, son las motivaciones las que conducen hacia el emprendimiento: independencia, reconocimiento, aprendizaje y roles (Scheinberg y MacMillan). Se debe señalar que dentro de la independencia se observan factores como: el miedo inminente a la pérdida de empleo o sustento (empuje) y deseo de aprovechar una oportunidad a pesar del riesgo de la pérdida de fuente de ingreso segura (atracción).

En el año 2002, con el fin de fortalecer el Sistema de Incubación de Empresas de Ecuador y con el auspicio de la CAF, el MICIP adquirió el modelo de Incubación de empresas de base tecnológica de Antioquia, para la creación de 3 incubadoras en el país: INCOVAL Guayaquil, Agencia Desarrollo Empresarial Loja, Incubadora de Empresas de Pichincha, cuyo denominador común es la suma de esfuerzos públicos, privados y académicos para el impulso al emprendimiento.

\section{- Objetivos}

\section{- Objetivo General}

Identificar y medir la actitud emprendedora de los estudiantes de la UTE que le permita establecer estrategias de promoción, comunicación, capacitación e impulso hacia el emprendimiento.

\section{○ Objetivos Específicos}

- Evaluar el perfil emprendedor que identifica a los estudiantes de la Universidad Tecnológica Equinoccial.

- Conocer la relación entre variables demográficas (edad, sexo, estado civil) de los estudiantes y su actitud hacia el emprendimiento.

- Identificar el proceso para la promoción, comunicación y capacitación de los estudiantes con perfil emprendedor.

- Identificar los aspectos que inciden en los estudiantes para tomar la decisión de establecer una empresa o negocio.

- Poner en conocimiento de la Universidad información que contribuya a promover y apoyar el desarrolloy fortalecimiento del emprendimiento en sus estudiantes.

\section{- Justificación}

Vivimos la era del conocimiento y del cambio que exige la globalización, la universidad para lograr posicionarse en este entorno debe emplear nuevas metodologías para la formación de los estudiantes, que permitan ofertar al mercado laboral profesionales con visión generadora de empleo y no de empleados, y la formación de una sociedad que verdaderamente se interese por reducir el desempleo y la pobreza.

\section{Tsafiqu}


El proyecto pretendió conocer la situación del estudiantado de la UTE, para que a partir de ello se establezca las estrategias de promoción, comunicación, capacitación e impulso al emprendimiento y lograr mantener la excelencia en todos los aspectos que a la Institución le corresponde por su trayectoria de servicio a la comunidad.

\section{- Contexto}

Inicialmente la palabra emprendimiento fue utilizada para referirse a los aventureros como Colón que venían al Nuevo Mundo sin saber con certeza que esperar, él mantuvo su obsesión de la existencia de una ruta más corta a las Indias y no se rindió a las continuas negativas de diversos reyes en Europa, hasta que encontró alguien que lo apoyara, al que convenció de la existencia de esta oportunidad y de que él era el hombre para llevarla a cabo, esta actitud es la que caracteriza al emprendedor, una actitud hacia la incertidumbre. Su origen hace referencia al término francés entrepreneur (pionero).

Fue Joseph Schumpeter, profesor de Harvard quien utilizó por primera vez el término emprendedor, para referirse a los individuos que con sus actividades generan inestabilidad en los mercados. Esta forma de pensamiento se contrapone a la escuela austriaca, la que señala que los emprendedores logran mejorar y hacer más eficientes la red comercial, anulando las turbulencias y creando nueva riqueza.

Los ejecutivos tienen su propia percepción sobre el espíritu emprendedor, lo describen como "innovador, flexible, dinámico capaz de asumir riesgos creativo y orientado al crecimiento"”.

La prensa por su parte lo define como la capacidad de iniciar y operar nuevas empresas. Se ha producido y se está produciendo un proceso por el cual las viejas empresas e industrias están siendo reemplazadas por las nuevas, liderado por los que se conocen como la Generación Emprendedora o simplemente la Generación-E,

\footnotetext{
'Raphael Amit. 1997. Charla intra empresariado. Sydney. Australia.
}

caracterizada por el pensamiento “obsesivo”, razonan y actúan frente a una oportunidad y ejercen un liderazgo balanceado.

Diferentes estudios muestran que la Generación-E es gente diversa y que están presentes en todas las áreas. Los empresarios y los intraemprededores, denominando asía aquellos que crean nuevas oportunidades para sus propias organizaciones, disfrutan su trabajo porque es estimulante, energético y significativo; porque inventan, adaptan, reconocen e impulsan nuevas ideas hasta transformarlas en nuevas oportunidades.

La fuerza más poderosa que está detrás de la creación de crecimiento económico y cambio social, es el espíritu emprendedor, representando la mayor expresión de democracia y la libertad por su capacidad de romper barreras. En la médula del proceso de emprendimiento esta el espíritu de innovación.

\section{- La actitud y el aprendizaje en el emprendimiento}

Es una forma de respuesta a alguien o a algo aprendida y relativamente permanente. La actitud es un elemento importante para la predicción de las conductas, y se ha definido como "reacción afectiva positiva o negativa hacia un objeto o proposición abstracto o concreto denotado". Las actitudes son aprendidas. Las actitudes raras veces son asunto individual; generalmente son tomadas de grupos a los que debemos nuestra mayor simpatía.

Las actitudes se componen de 3 elementos: lo que piensa (componente cognitivo), lo que siente (componente emocional) y su tendencia a manifestar los pensamientos y emociones (componente conductual).

Las emociones están relacionadas con las actitudes de una persona frente a determinada situación, cosa o persona. Entendemos por actitud una tendencia, disposición o inclinación para actuar en determinada manera. Ahora bien, en la actitud (preámbulo para ejercer una conducta), podemos encontrar varios elementos, 
entre los que se distinguirán los pensamientos y las emociones. Por ejemplo, en el estudio de una carrera, si la actitud es favorable, encontraremos pensamientos positivos referentes a ella; así como, emociones de simpatía y agrado por esos estudios. Las emociones son así ingredientes normales en las actitudes. Una vez formada, es muy difícil que se modifique una actitud, ello depende en gran medida del hecho de que muchas creencias, convicciones y juicios se remiten a la familia de origen. En efecto, las actitudes pueden haberse formado desde los primeros años de vida y haberse reforzado después. Otras actitudes se aprenden de la sociedad, como es el caso de la actitud negativa ante el robo y el homicidio; por último otros dependen directamente del individuo.

Hay veces que las actitudes pueden modificarse, lo cual obedece a diversos motivos. Por ejemplo, una persona puede cambiar de grupo social y asimilar las actitudes de éste. Lo que en mayor grado puede cambiar una actitud es la información que se tiene acerca del objeto.

En este contexto la teoría del aprendizaje señala que aprendemos actitudes del mismo modo en que aprendemos todo lo demás, estas teorías del aprendizaje conciben a las personas como seres primariamente pasivos, cuyo aprendizaje “depende del número y de la fuerza de los elementos positivos y negativos previamente aprendidos".

El entrenamiento clásico formal no es la única forma por el cual un estudiante universitario aprende, mucho de ese entrenamiento se completa con la experiencia que adquiere de su trabajo o en la red de contactos personales fuera del aula. Alan Gibb (1993) sostiene que los contenidos pedagógicos y las metodologías didácticas empleadas tradicionalmente fortalecen valores y habilidades que no animan al emprendedorismo.

A nivel universitario se debe establecer claramente el papel del formador de emprendedores y romper la tensión entre la teoría basada en la acumulación de grandes cantidades de información y la diseminación del conocimiento a cargo de un docente / entrenador y la práctica de reproducir en el aula, el contexto donde el emprendedor aprende como el empresario emprende.

No es solamente un simple cambio de enfoque, pues las universidades se han especializado en la enseñanza de habilidades para la resolución de problemas, sin considerar que este proceso está viciado de asunciones teóricas, donde se logra un acercamiento del alumno al conocimiento dentro de un proceso pasivo, en el que es presionado con tiempos limitados a comprender lo que se muestra y a que lo integre con lo aprendido, utilizando en muchas ocasiones la intuición o especulando para resolver los vacíos entre las teorías y la realidad. En 1984 el Babson College (USA) inició una serie de programas de Entrepreneurship, ellos consideran que no puede enseñarse para "ser empresario", pero pueden enseñarse las habilidades que un empresario necesita para tener el éxito.

Es importante integrar la investigación y enseñanza del Entrepreneurship con el mundo real de la dirección y los procesos de toma de decisiones empresariales. Si consideramos las recomendaciones de las escuelas anglosajonas asegurémonos que éstas se adecuen a las realidades culturales, tecnológicas y económicas de América Latina.

El aprendizaje de competencias empresariales se basa en actitudes emprendedoras y la dirección de la formación emprendedora del Siglo XXI que es fortalecer el desarrollo actitudinal para desarrollar competencias eficaces de dirección empresarial eficiente. Es necesario sincerar que emprender es la acción independiente de un individuo, equipo u organización para materializar una idea y que formar emprendedores es facilitar ese proceso. El desafío para los educadores universitarios, es poner el énfasis en actividades educativas para "Aprender haciendo" y aceptar que se está fracasando con los esfuerzos para "enseñar a emprender" y dar los primeros pasos por los procesos de facilitación

\section{Tsafiqu}


para "aprender a emprender".

\section{- Emprendimiento, Vínculo: Empresa, Educación y Gobierno}

Muchas veces la sociedad reclama a la universidad una respuesta acorde a las necesidades sociales relativas al desarrollo empresarial y su consecuente creación de empleo, allí surgen ciertas interrogantes ¿Nos hemos ceñido demasiado a la formación del conocimiento y no desarrollamos nuestra capacidad para transferirlo adecuadamente a los intereses y necesidades específicas regionales? ¿Transitamos por un camino que no da los resultados esperados? ¿No comprendimos la naturaleza sustantiva de la demanda social y empresarial?

Los expertos sostienen que la universidad produce recursos humanos para las empresas y se olvidan la formación de humanos con recursos capaces de crear sus propias empresas o mejorar lo que ya existe ampliando la riqueza de sus respectivos contextos sociales; la universidad sigue formando profesionales con habilidades para los negocios, dejando pasar la oportunidad de consolidar sus habilidades empresariales.

La educación es factor clave para explicar el proceso de desarrollo de nuevos emprendimientos, los que generan efectos positivos sobre el progreso económico, empleo y dinamismo de la economía. De ella dependerá el surgimiento de empresas basadas en el conocimiento y la tecnología. En los últimos años, algunos gobiernos como Alemania, Escocia e Irlanda, han puesto en marcha diferentes proyectos e iniciativas, encaminadas a desarrollar el potencial existente en las universidades e institutos de investigación para la conformación de empresas de este tipo². De igual forma se ha visto un compromiso de las universidades de Latinoamérica y otras partes del mundo, con la incorporación del Entrepreneurship en la formación universitaria y el desarrollo de las capacidades

2 POSTIGO, Sergio, TAMBORÍN, Ma. Fernanda, “Educación y Entrepreneurship en Argentina: El caso de la Universidad de San Andrés". emprendedoras de los estudiantes (Postigo y Tamboríni, 2002).

Al igual, Klandt (1993) señala, que las capacidades emprendedoras no son innatas, sino que pueden ser adquiridas y por lo tanto, pueden descubrirse y guiarse mediante un proceso de aprendizaje dirigido, no solo hacia la transferencia del conocimiento teórico y de aspectos instrumentales de la planificación y la gestión, sino también al desarrollo de competencias, habilidades, actitudes y valores, empleando herramientas de aprendizaje activoparticipativas, basado en la resolución de problemas y experiencias reales, como juego de roles, visitas y charlas con emprendedores, simulaciones de negocios, planes de negocios, entre otros.

Los aspectos actitudinales sostienen las habilidades empresariales a largo plazo y los programas de entrenamiento deben apuntar a que los estudiantes logren autonomía para formarse visiones personales y tomar decisiones de largo alcance, aspecto diferenciador entre el comportamiento empresario y la mera habilidad para administrar negocios propios o ajenos.

El emprendedor es el fruto de una cultura, y es responsabilidad social de la universidad profundizar y promover factores que faciliten los eventos emprendedores entre los estudiantes, y creen habilidades para que el alumno transfiera lo aprendido en el contexto del aula a otros contextos donde él actúa.

Uno de los gobiernos latinoamericanos que ha dado gran impulso a este tema es el chileno, en el cual el Ministerio de Economía propuso como política de Estado el tema de Emprendimiento, y la creación del Programa Público Privado de Fomento al mismo, en el año 2002, como una instancia de diálogo entre agentes públicos, inversionistas, emprendedores y personas vinculadas al mundo académico. Hoy el emprendimiento es el punto focal en la estrategia productiva del país, vinculando estrechamente a la empresa, la educación y el gobierno, donde el eje verdadero de articulación social y de desarrollo nacional es la educación y la empresa se transforma

\section{Tsafiqu}


en un actor con responsabilidad social. ${ }^{3}$

Chile dispuso que esta asignatura cruce transversalmente los límites definidos por las fronteras disciplinarias y que busca el desarrollo de las habilidades y conocimientos para identificar y resolver problemas en los cuales la aplicación tecnológica significa un aporte a la calidad de vida de las personas, en donde el aprendizaje es responsabilidad profesional de los docentes.

\section{- El emprendimiento en la UTE}

La UTE, a partir del año 2003 fortalece su atención hacia el emprendimiento y en el cambio del paradigma vigente, para evolucionar de la preparación de profesionales empleados, hacia profesionales creadores de empresas; a raíz de su interés y su participación en la Incubadora de Empresas de base tecnológica de Pichincha-Emprender. A partir de estas experiencias se crea el SEAFE (Sistema Ecuatoriano de apoyo a la formación de Emprendedores), que actualmente incluye trece universidades a nivel nacional.

El Sistema incluye, primero el Programa de Capacitación a los Docentes, segundo, la creación de la Cátedra de Emprendimiento, tercero, diseño de material didáctico de apoyo a la cátedra, conformado por veinte (20) casos y diez (10) videos de emprendedores ecuatorianos que complementan el proceso de enseñanzaaprendizaje del emprendimiento.

En la actualidad la UTE ha incorporado la materia Desarrollo de Emprendedores en todas sus Carreras a nivel central y de sus Campus en Santo Domingo de los Colorados y Salinas. Se han formado alrededor de 120 docentes en metodologías activo-participativas que contribuyen a cómo aprender a emprender.

Se realizó en dos fases: La primera tuvo como propósito despertar el espíritu emprendedor en los participantes y la segunda, reforzar el desarrollo de planes de negocios. En este proceso Educación para el Emprendimiento, Ministerio de Educación, Chile, 2004. han participado más de 2000 estudiantes. Con el fin de continuar impulsando el emprendimiento al interior de la universidad, se realizó Primer Concurso de empresas Exitosas, dirigido principalmente a los estudiantes, abierto a la participación de docentes.

\section{Materiales, Métodos y Procedimientos}

La Unidad de Análisis estuvo conformada por los estudiantes de la UTE de todas las jornadas de estudio y de ambos géneros. Un total de 6.250 estudiantes reportados en el registro de la universidad conforman la Población (N). Se seleccionaron 245 estudiantes de ambos sexos en las tres jornadas mencionadas que conformaron la Muestra (n), no probabilística intencionada, estratificada, proporcional al tamaño de las Facultades y Programas Académicos. Cuando el número de casos fue cero, se decidió tomar un número de 5 estudiantes, para que la Escuela respectiva no quedara eliminada del estudio. A continuación se presenta la distribución de la muestra:

$\checkmark \quad$ Artes: Arquitectura: (9) y Modas (5)

$\checkmark$ Economía: Comercio Exterior (31), Ingeniería de Empresas (19), Marketing (23), Ing. Financiera (1)

$\checkmark$ Ingeniería: Industrial (6), Alimentos (6), Informática (15), Mecatrónica (5), Petróleos (6), Textil (6).

$\checkmark \quad$ Medicina: Emergencias (5), Medicina (4)

$\checkmark$ Sociales: Diseño Gráfico (5), Publicidad (15), Parvularia (5), Relaciones Públicas (15).

$\checkmark \quad$ Turismo: Gastronomía (25), Turismo (16), Hotelería (23)

Para la obtención de la información se utilizó el Cuestionario de Auto Evaluación de las conductas emprendedoras, desarrollado y probado por el Programa Formación de Multiplicadores en Emprendimiento de INCUBATEC (Incubadora de Negocios de 
Temuco, Chile). El cuestionario consta de 55 declaraciones breves, de las cuales los encuestados seleccionaron una respuesta, marcando el número correspondiente en una escala de 1 a 5.

Se realizó el vaciado de respuestas en el cuadro correspondiente, aplicando las instrucciones para completar las puntuaciones de las CCE, sumadas arrojaron la puntuación total de cada uno. Se aplicó igualmente un procedimiento para la corrección de la puntuación.

Para la obtención de los resultados, los cuales se reflejan en un perfil, se trasladaron las instrucciones del vaciado de respuestas al programa Excel, lo que permitió establecer el perfil de los encuestados.

\section{- Trabajo de campo}

La aplicación del cuestionario fue realizada durante el mes de noviembre del año pasado. Se procedió al vaciado de los datos en el programa indicado, los cuales se incluyeron por una parte en cuadros de salida y gráficos, en números absolutos y porcentajes y por otra, en la hoja de vaciado de respuestas, las puntuaciones correspondientes. Posteriormente, se procedió al análisis e interpretación de los resultados.

\section{Resultados y Discusión}

La presentación de éstos se inicia con información general: Distribución de las unidades muestrales por Facultad y Jornadas, los Niveles (Facultades y Escuelas), Género y Estado civil.

\section{- Distribución de los informantes por Facultad y Jornada}

La distribución de las unidades muestrales fueron en su orden: mañana, noche, en tercera instancia, sesión de la tarde. Predomina el número de estudiantes en las sesiones de la mañana y la noche en todas las Facultades.

\section{- Distribución por Niveles (Facultades. Escuelas).}

La distribución de los datos por Niveles, en Facultades y Escuelas. Permite resaltar que Comercio Exterior, Gastronomía y Marketing aportaron el $13 \%, 10 \%$ y $9 \%$ respectivamente de Informantes, seguido de empresas con el $8 \%$, Turismo y Mecatrónica con el $7 \%$ y Relaciones Públicas con el $6 \%$ en tanto que las demás aportaron se movieron en el $2 \%$

\section{- Distribución por Género}

Por género, predominó el masculino con el 53\% de los informantes, (131), frente al 47\% de femenino (114). Por Facultades se mantiene, en términos generales, la misma distribución proporcional.

\section{- Distribución por estado civil}

Para la variable Estado civil, los datos muestran que predomina ampliamente los solteros con 223 casos, (91\%), diez y siete solteros (7\%) y un $1 \%$ divorciados o en unión libre. Es un resultado lógico y similar al de otras universidades.

\section{- Perfil emprendedor de los estudiantes}

A continuación se presentan los resultados respecto a las variables relacionadas con el perfil emprendedor de los estudiantes de la UTE.

Los datos arrojados del Cuestionario de Auto Evaluación de las Conductas Emprendedoras ${ }^{4}$, revelan el perfil emprendedor de los estudiantes de la UTE. Éstos valores se categorizan así:

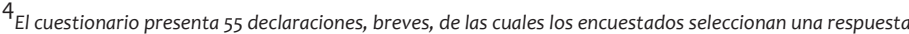
en una escala de1 a 5 , en donde (1) indica: Nunca es cierta la declaración, (2), raras veces, (3) algunas veces, (4), usualmente y (5) siempre 
- $\quad$ De 5 a 9, perfil bajo;

- $\quad$ De 10 a 15, buen perfil;

- $\quad$ De 16 a 20 muy buen perfil, y;

- $\quad$ Entre 21 y 25 , excelente perfil.

\section{- Conductas}

\section{Gráfico No1}

\section{Conductas por Género}

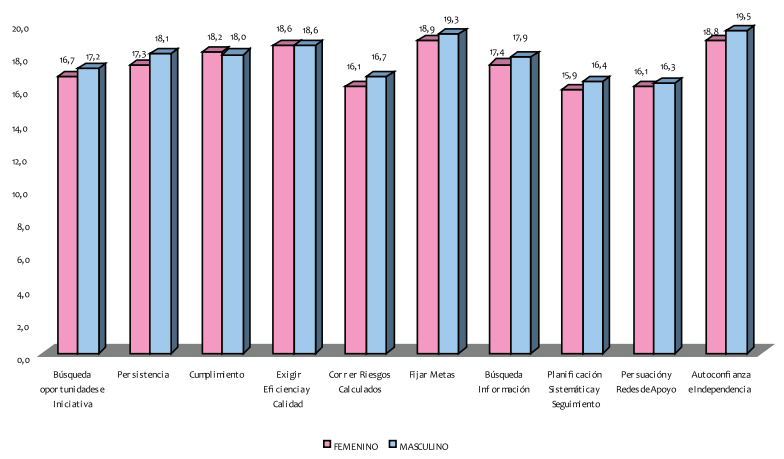

Las diez conductas se ubican en el rango de 16 a 20, tanto para las mujeres como para los hombres, que se considera como muy buenos perfiles de emprendedores. Las diferencias entre uno y otro son mínimas si especificamos, podemos decir que la autoconfianza e independencia (19.5) y el fijar metas (19.3) logran los valores más altos, en tanto que en las mujeres, sobresalen el fijar metas (18.9) y auto confianza e independencia (18.8).

Los resultados permiten observar que los estudiantes de la UTE dentro del perfil de emprendimiento presentan una elevada auto confianza e independencia sobre las demás conductas, seguido de la fijación de metas, con lo que podemos inferir que nuestros estudiantes tanto hombre como mujeres se plantean objetivos y con confían en sus capacidades de logro. Por el contrario, sin salirse de este rango de buen perfil, tenemos que es menos usual que ellos planifiquen sus metas, busquen redes de apoyo para lograrlas y calculen los riesgos a los que puedan enfrentarse.

\section{- Por Jornadas}

Las conductas auto confianza e independencia, el fijar metas y el exigir eficiencia y calidad, se ubicaron en la frontera entre muy bueno y excelente conducta y entre el limite de buen perfil y muy buen perfil se ubica la planificación sistemática y seguimiento, así como el correr riesgos calculados.

\section{Gráfico No2}

\section{Conductas por Jornadas}

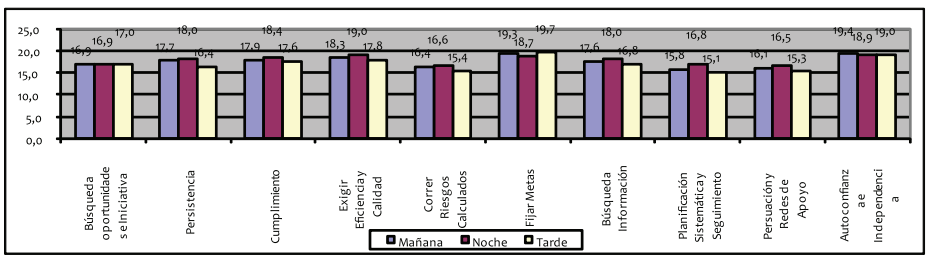

Se da menor importancia a la planificación y cuando se trata de arriesgar no se está considerando hasta que punto hacerlo y cómo enfrentarlos adecuadamente a fin de evitar un impacto negativo sobre nuestros proyectos y acciones. Esto permite inferir que la actitud hacia el emprendimiento en los estudiantes de la mañana, tarde y la noche es muy parecido en todas las jornadas.

Se pretendió conocer si había diferencias en las conductas considerando las Jornadas de estudio, ya que los estudiantes en las jornadas de día, generalmente no asumen el costo de sus estudios, en cambio los de la jornada nocturna, se presume que asumen los costos de sus estudios. Los datos no presentan diferencias promedias significativas en las conductas entre las jornadas. 


\section{- Por Facultades}

\section{Gráfico No 3}

\section{Conductas por Facultad}

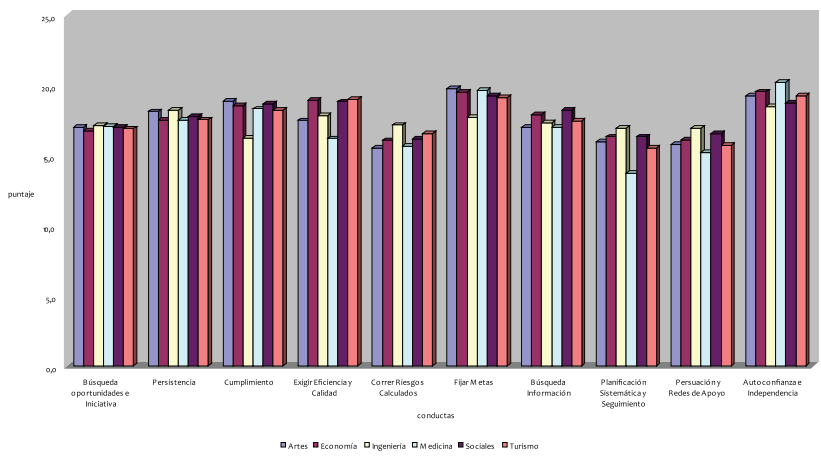

En los perfiles por facultades, los datos para conductas examinadas 5 se ubican dentro del rango de 16 a 20, (muy buen perfil) y en el rango 10 a 15 (buen perfil), es decir algunas facultades se están en los rangos altos. Los estudiantes en general presentan conductas y actitudes, favorables hacia el emprendimiento, lo cual permitirá que las acciones que la UTE proponga a este respecto, den una respuesta favorable por parte de los estudiantes.

\section{- Por Curso}

\section{Gráfico No4}

\section{Conductas por Curso*}

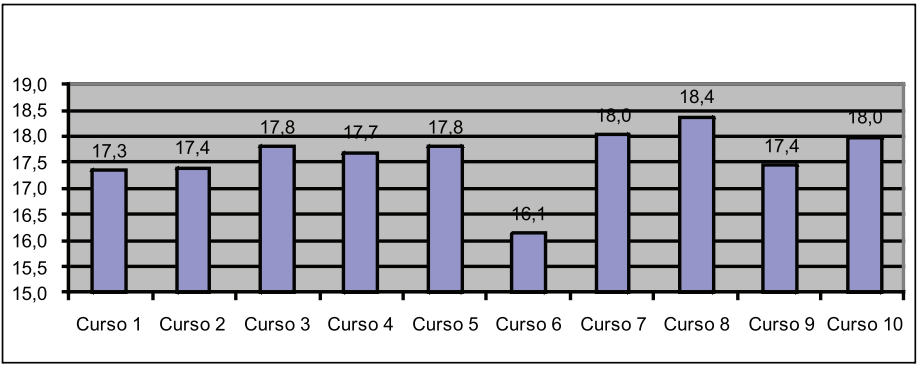

* Se tomaron promedios para este Gráfico.

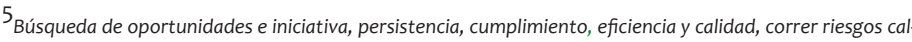
culado, fijar metas, búsqueda de información, planificación, sistemática y seguimiento, persuasión y redes de apoyo, y, auto confianza e independencia
}

Los perfiles actitudinales de los estudiantes mostraron el rango de muy buenos perfiles. Los estudiantes del sexto curso, se ubican al inicio de este rango (16.1), en comparación con los demás cursos, que se ubican entre 17.0 y 18.5, manteniéndose aún dentro del rango indicado. Con mayor promedio están los niveles más altos de las carreras, séptimo a décimo, observándose en el noveno nivel un promedio menor. Los resultados demuestran que los cursos, desde el inicio hasta la finalización de los estudios, cuentan con altos niveles de aceptabilidad de acciones de la universidad hacia el emprendimiento por parte de los estudiantes. Para conocer la distribución de los diferentes tipos de conductas hacia el emprendimiento, los cuales dan como resultado el tipo de perfil emprendedor, se organizaron las respuestas para cada una de las Facultades, lo que permitió tener una visión individualizada y a la vez panorámica del perfil o perfiles más representativos de los estudiantes / facultad y universidad. Los resultados se presentan haciendo un breve análisis por conducta / facultad y al final de éste un análisis total.

\section{- Por Estado Civil}

\section{Gráfico No $5^{*}$}

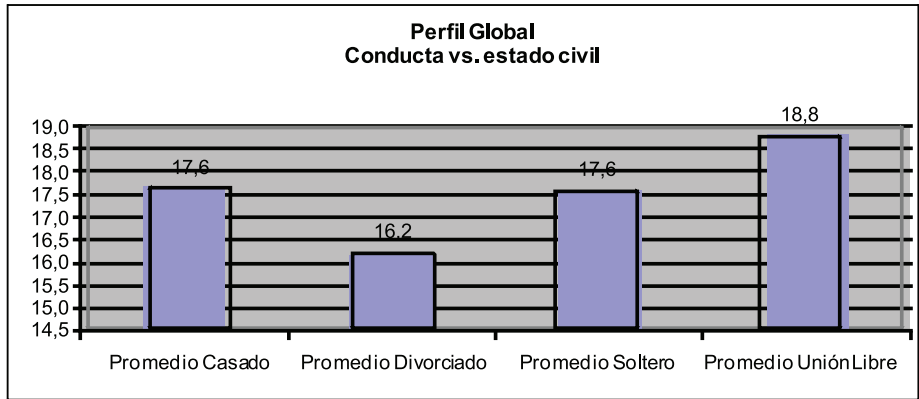

* Se tomaron valores promedio

La relación entre las conductas y las variables sociodemográficas consideradas, indicó una situación favorable hacia el emprendimiento por parte de los estudiantes de los cuatro estados, mayormente en los casados y en unión, libre frente a los divorciados y solteros. Los resultados arrojados indican que ha sido un acierto por parte de la Universidad el fortalecer este componente en la 
formación académica. Si bien es cierto que entre los divorciados las conductas arrojaron resultados menos favorables, en general existe una actitud positiva hacia el emprendimiento con la variable estado civil.

\section{- Por Edad}

\section{Gráfico No 6}

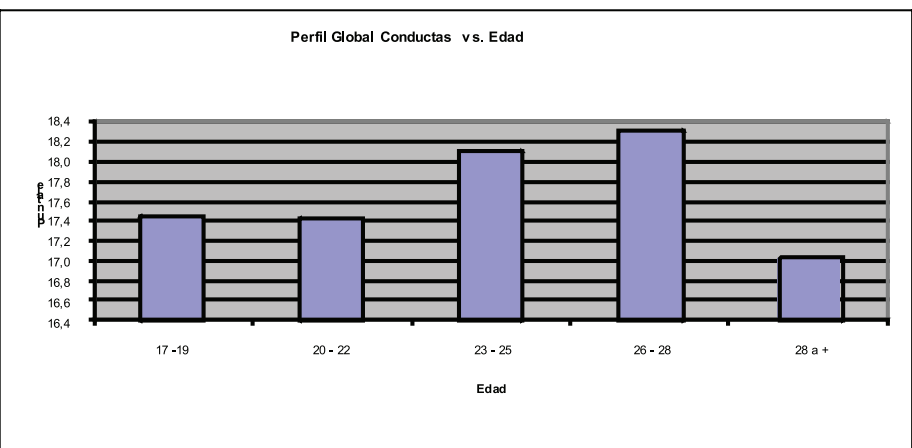

En las conductas: Persistencia, eficiencia y calidad, riesgos, búsqueda de información, planificación sistemática y seguimiento, persuasión y redes de apoyo, y auto confianza e independencia, los datos arrojaron que los estudiantes entre las edades de 23 años y 28 en adelante son los que presentaron homogeneidad en aquellas conductas. Los autores no encontraron información de otros estudios que les permitiera hacer inferencias, así que dejamos estos datos encontrados para futuras investigaciones que incluyan esta relación.

\section{- Qué necesita para Emprender}

Un último componente incluido en este estudio lo constituyeron seis que fueron ponderados (mínimo 1, máximo 6): Motivación, dinero, actitud, conocimiento, capacidades y tiempo. Se dividieron en las tres jornadas de estudio y el géneros.

Analizados los resultados se encontró que las necesidades mencionadas por los estudiantes de sexo masculino (ponderación máxima) para emprender negocios fueron:

- Jornada matutina: Dinero (31,3\%) y tiempo (37,6\%);

- Jornada de la tarde: Motivación, actitud y tiempo (32,5\% para cada uno);

- Jornada de la noche: Dinero (32,7\%) y tiempo (30,9\%) respectivamente.

En el sexo femenino la distribución fue la siguiente:

- Jornada de la mañana, dinero con el 37,7\% y actitud con el $23,0 \%$;

- Jornada de la tarde, dinero (53,8\%) y tiempo (30,8\%),

- Jornada de la noche: $53,8 \%$ mencionaron el dinero y tiempo el $30,8 \%$.

Se consideraron y resaltaron los factores que mayor mención hicieron los estudiantes sobre las necesidades sentidas como críticas para el emprendimiento fueron el dinero, el tiempo y las actitudes hacia el emprendimiento y son consideradas como barreras sentidas para el Star-up de nuevas empresas o negocios.

\section{Conclusiones y Recomendaciones}

\section{Conclusiones}

La importancia de fortalecer este nuevo enfoque académico para cambiar la actitud y conducta de los futuros profesionales, que les permita convertirse ya no en empleados sino en creadores y desarrolladores de su propia empresa, es una necesidad imperante en el mundo actual y futuro.

Así la UTE, a partir del año 2003 dio inicio a una estrategia hacia el desarrollo y fortalecimiento del emprendimiento entre

\section{Tsafiqu}


sus estudiantes y docentes, en el entendido de que representa una nueva oportunidad que conlleva al cambio en el aprendizaje y ejercicio de los nuevos profesionales creadores de empresas y auto empleadores.

Entre las diferentes actividades establecidas, como la creación de la Cátedra de Emprendimiento, la formación de un grupo de docentes, el impulso entre la comunidad académica a participar activamente en proyectos cuyo centro de atención es el emprendimiento.

Se aceptó por parte de la UTE la propuesta de los Profesionales del ITT autores de esta investigación cuyo objetivo fue el de identificar y medir la actitud emprendedora de los estudiantes de la UTE, para diseñar estrategias de promoción, comunicación, capacitación e impulso al emprendimiento.

Luego del análisis de los factores actitudinales y conductuales de los estudiantes hacia el emprendimiento y su asociación con otras variables socio-demográficas y la identificación de las necesidades sentidas para emprender negocios permitieron referir las siguientes conclusiones:

1. Los resultados arrojados demostraron que existe actitudes y conductas altamente positivas hacia el emprendimiento dentro del estudiantado.

2. Existe actitudes en los docentes y estudiantes, así como en los directivos de apoyo a las estrategias que conlleven a fortalecer las actividades que conduzcan a fomentar una cultura emprendedora

3.Un perfil, de acuerdo a los valores de los indicadores del Test utilizado, altamente positivo entre los estudiantes tanto en uno como en otro género.

4.Se puede inferir que la Universidad está acertando en sus estrategias hacia un enfoque de formación académica que conlleve al emprendimiento en sus estudiantes y motivación en los docentes. Muestra de ello son los Talleres que se han impartido a los docentes en toda la universidad, la inclusión de la Asignatura de Emprendimiento en todas las Facultades, la promoción de concursos y ferias para promoverlo.

5.Un marcado y progresivo interés en los estudiantes por encontrar fuentes y recursos económicos que les permita presentar proyectos con enfoque emprendedor, en especial a los que han demostrado actitudes y conducta altamente innovadoras.

6.En cuanto a la variable género frente al emprendimiento, se observó un gran interés y actitudes altamente positivas hacia el mismo sin encontrar deferencias significativas de estas actitudes analizadas tanto en hombres como en mujeres. A igual conclusión se llegó en cuanto a la variable 'jornadas académicas.

7. Por Facultades, Institutos y Programas los resultados arrojados por el Test indicaron que existe muy buena actitud y conducta hacia el emprendimiento y cada una de ellas está sobre el promedio de 17 puntos.

8.La relación entre estado civil de los estudiantes en las tres jornadas, mostró que es mayormente positiva entre los casados y en unión libre frente a los divorciados y solteros.

9.En cuanto a la variable edad predominó que los estudiantes entre las edades de 23 a 28 años conductas positivas hacia el emprendimiento.

10. Respecto a la posición de los estudiantes, tanto en las diferentes jornadas como por género sobre las dificultades para tomar decisiones de lanzarse hacia el "Star-up" de negocios empresas propias, los resultados indicaron que dinero, tiempo, motivación y actitudes fueron consideradas 
comolas más críticas. Se consideraron y resaltaron los factores que mayor mención tuvieron, que según las apreciaciones de los estudiantes son fundamentales y críticas para emprender en un nuevo negocio, las otras razones, conocimiento y capacidades son importantes considerarlas, ya que es el sentir del estudiante que dispone de estas cualidades y no son barreras determinantes para iniciar una nueva empresa.

\section{Recomendaciones}

1. Que la Universidad continúe con la estrategia que se ha trazado para lograr la consolidación del Emprendimiento en sus estudiantes y docentes, por la disposición y por la fertilidad del terreno que es posible apreciar en este estudio.

2. Es importante también continuar con programas, eventos de capacitación y actualización en docentes y estudiantes que incentiven y aprovechen la capacidad, el espíritu emprendedor que se observa, existe en los estudiantes.

3. Integrar el emprendimiento y la investigación, con lo cual se logrará encontrar ideas innovadoras, congruentes con las características y requerimientos del país.

\section{Bibliografía Consultada}

1. Bossidy, Larry y Charan, Ram. El arte de la negociación en los negocios. Editorial Aguilar. Buenos Aires, Argentina. 2002.

2. Cerda, E. Herder Una Psicología de hoy. Editorial Norma. Bogotá. Colombia, 1999.

3.Cortes, Patricio. Director Ejecutivo, Centro para el Emprendimiento y la Innovación (CEI/UDD) Dirección de Comunicaciones Escuela de Postgrado Facultad de Economía y Empresa Universidad Diego Portales, Chile.

4.Freire, Andy-Pasión por emprender: De la idea a la cruda realidad. Editorial Aguilar. Buenos Aires, Argentina. 2004.

5. Fisher, Robert. Ury, William y Patton, Bruce. Si.... De acuerdo. Cómo negociar sin ceder. Editorial Norma, novena edición. Bogotá, Colombia, 1999.

6. Hernández, Roberto, Fernández, Carlos y Baptista, Pilar. Metodología de la Investigación. Editorial Mc Graw-Hill. ·3a. Edición. México. 2004.

7. Krause, Martín. Economía para emprendedores. Editorial Aguilar. Buenos Aires, Argentina. 2004.

11. Vejarano, Gilberto. Apuntes de metodología de investigación. UTE. Dirección General de Postgrados. Quito. Ecuador. 2006

12. http://www.justoahora.com/nws/v15.htm\#2

13. http://www.postgradosingenieriaynegocios.cl/noticias/ educacion_y_emprendimiento/

\section{El Investigador}

\section{Dr. Gilberto Vejarano Monroy} gvejaran@ute.edu.ec

Licenciado en Sociología y Antropología, Universidad Nacional de Colombia, 1966

M.Sc. Sociología, lowa State University. lowa. USA, 1972

Ph.D. Comunicación, Florida State University. USA , 1979 